\title{
Dual Description of Supergravity MacDowell-Mansouri Theory
}

\author{
H. García-Compeán ${ }^{a *}$, J. A. Nieto ${ }^{b \dagger}$, O. Obregón ${ }^{c \ddagger}$ and C. Ramírez ${ }^{d \S}$ \\ ${ }^{a}$ Departamento de Física, Centro de Investigación y de Estudios Avanzados del IPN \\ P.O. Box 14-740, 07000, México D.F., México \\ ${ }^{b}$ Facultad de Ciencias Físico-Matemáticas, Universidad Autónoma de Sinaloa \\ C.P. 80000, Culiacán Sinaloa, México \\ ${ }^{c}$ Instituto de Física de la Universidad de Guanajuato \\ P.O. Box E-143, 37150, León Gto., México \\ ${ }^{d}$ Facultad de Ciencias Físico Matemáticas, Universidad Autónoma de Puebla \\ P.O. Box 1364, 72000, Puebla, México
}

(July 26, 2021)

\begin{abstract}
In the context of field theory two elements seem to be necessary to search for strong-weak coupling duality. First, a gauge theory formulation and second, supersymmetry. For gravitation these two elements are present in MacDowell-Mansouri supergravity. The search for an "effective duality" in this theory presents technical and conceptual problems that we discuss. Nevertheless, by means of a field theoretical approach, which in the abelian case
\end{abstract}

\footnotetext{
*Present Address: School of Natural Sciences, Institute for Advanced Study, Olden Lane, Princeton NJ 08540 USA. E-mail: compean@sns.ias.edu

${ }^{\dagger}$ E-mail: nieto@uas.uasnet.mx

${ }^{\ddagger}$ E-mail: octavio@ifug3.ugto.mx

$\S$ E-mail: cramirez@fcfm.buap.mx
} 
coincides with $S$-duality, we exhibit a dual theory, with inverted couplings. This results in a supersymmetric non-linear sigma model of the FreedmanTownsend type.

PACS numbers: 04.60.-m, 04.65.+e, 11.15.-q, 11.30.Ly 


\section{INTRODUCTION}

Duality in supersymmetric gauge field theory and superstring theory is a matter of great deal of recent investigation. It reveals new profound insights in the perturbative as well as in the nonperturbative sectors of these theories (for recent reviews see [1,2]). In perturbative superstring theory, for instance, $T$-duality and in general, mirror symmetry, gives a surprising new view of space-time physics revealing the existence of a minimal length. It also provides a powerful tool to study stringy phenomena of space-time physics, such as the idea of worldsheet equivalence of non-smooth space-time transitions whose prime example is, of course, the change in the space-time topology. Furthermore, the strong-weak coupling duality or $S$-duality has become a most important technique to study non-perturbative aspects of field theory and string theory (see [1,2]). It turns out that $S$-duality is a fragile property in the sense that the absence of supersymmetry can spoil it. In fact, there are known examples of non-supersymmetric field theories possesing the property of S-duality [4]. However as far as duality is well understood in the presence of supersymmetry, we expect that in the case of MacDowell-Mansouri (MM) theory, supersymmetry will improve

the S-dual description. The absence of supersymmetry implies also the absence of the stability of the spectra of masses under the renormalization group flow of BPS states [3]. In superstring theory, duality also shows the equivalence among different types of perturbative string theories and indicates a strong evidence of an emerging underlying theory known as $M$-theory [2]. On the other hand, for $\mathrm{N}=2$ supersymmetric gauge theories in four dimensions, Seiberg and Witten found that a strong-weak coupling 'effective duality' can be defined on its low energy effective theory for the cases pure and with matter [5].

Exploring analogies and generalizations of that genuine $S$-duality, people obtained other kinds of "dualities". For instance, in non-supersymmetric non-abelian gauge theory, it is possible to define a "field theory duality" by constructing explicitly the dual action to YangMills action with a theta term, following the usual Roček-Verlinde procedure [6] 8]. The resulting dual action is described by a non-linear "massive" sigma model of the type worked 
out in [9]. In particular, in [7], the obtained (local gauge invariant) dual variables were used by the authors to reproduce the large- $N$ limit of $\mathrm{SU}(N)$ partition function gauge theory in two-dimensional tori. In three dimensions, for $\mathrm{SU}(2)$, the dual theory is shown to be very close to gravity theories and their moduli space of solutions.

Following these ideas, in Refs. [10, 11] (and reviewed in [12]) it was shown that the MacDowell-Mansouri gauge theory of gravity in four dimensions 13, 14 does admit a dual description in the sense of [7]. This leads to a dual theory of gravity with the structure of a generalized non-linear sigma model. It is important to mention that our approach does not concern the origin of the theory, it could be seen as an effective theory, for which renormalizability has not to be discussed. Nevertheless, the theory could be also considered as an elementary theory, and in this case its quantization is an important issue. Thus one would like to be able to find an "effective gravitational duality", in the sense that Seiberg and Witten have found for standard gauge field theories. It is not clear if such a program would work for gravitation. However, it seems to us worthwhile to pursue this task. In the first place, we work with the MacDowell-Mansouri formulation, a gauge theory of gravity. The other element which seems to be necessary is supersymmetry. So, a natural next step is to consider the MM-supergravity version. In this paper we work out its dual description in the same sense of Refs. [10 12]. We are aware that a more rigorous definition of an "effective gravitational duality" would need a way to define a low energy effective theory [5], among other requirements. This task presents particular difficulties, by example, one needs to preserve the gauge theory structure of the MM-theory when coupled to matter [15]. In order to find the dual description of the MM-supergravity, in section II we present this theory and its (anti) self-dual versions. In section III the dual action is obtained from a parent action. Section IV is devoted to discussion. An alternative derivation of the dual action of MM-supergravity theory, showing explicitly the formalism of calculus in superspace, is presented in the appendix. 


\section{MACDOWELL-MANSOURI SUPERGRAVITY}

The theory of MacDowell-Mansouri is a gauge theory in $3+1$ dimensions, with the antide Sitter $\mathrm{SO}(3,2)$ gauge group [13,14. After breaking the original gauge group to $\mathrm{SO}(3,1)$, the resulting gauge theory leads to the Einstein-Hilbert action, cosmological constant term and Euler topological invariant.

The supersymmetric version of MM theory can be constructed simply by promoting the $\mathrm{SO}(3,2)$ gauge fields to those corresponding to the supergroup Osp(1|4). In particular the gauge potential $\mathcal{A}_{\mu}{ }^{A}$ is a $\operatorname{Osp}(1 \mid 4)$ - Lie algebra valued potential. The corresponding field strength is given by

$$
\mathcal{F}_{\mu \nu}{ }^{A}=\partial_{\mu} \mathcal{A}_{\nu}{ }^{A}-\partial_{\nu} \mathcal{A}_{\mu}{ }^{A}+\frac{1}{2} f_{B C}^{A} \mathcal{A}_{\mu}{ }^{B} \mathcal{A}_{\nu}{ }^{C},
$$

where $f_{B C}^{A}$ are the structure constants of the super Lie algebra Osp(1|4). The field strength $\mathcal{F}_{\mu \nu}^{A}$ can be decomposed into three terms corresponding to the three generators $S_{A}=\left(S_{a b}, P_{a}, Q_{i}\right)\left(\right.$ with $\left.P_{a}=S_{4 a}\right)$ of $\operatorname{Osp}(1 \mid 4)$ as

$$
\mathcal{F}_{\mu \nu}^{a b}=F_{\mu \nu}^{a b}+\Sigma_{\mu \nu}^{a b}+\Theta_{\mu \nu}^{a b}, \quad \mathcal{F}_{\mu \nu}^{i}=F_{\mu \nu}^{i}+\Sigma_{\mu \nu}^{i}, \quad \mathcal{F}_{\mu \nu}^{a}=F_{\mu \nu}^{a}+\Sigma_{\mu \nu}^{a},
$$

where

$$
\begin{gathered}
F_{\mu \nu}^{a b}=\partial_{\mu} A_{\nu}^{a b}-\partial_{\nu} A_{\mu}^{a b}+\frac{1}{2} f_{c d e f}^{a b} A_{\mu}^{c d} A_{\nu}^{e f}, \\
\Sigma_{\mu \nu}^{a b}=2 f_{4 c 4 d}^{a b} A_{\mu}^{4 c} A_{\nu}^{4 d} \\
\Theta_{\mu \nu}^{a b}=\frac{1}{2} f_{i j}^{a b} A_{\mu}^{i} A_{\nu}^{j}, \\
\sum_{\mu \nu}^{a}=\frac{1}{2} f_{i j}^{4 a} A_{\mu}^{i} A_{\nu}^{j}, \\
\Sigma_{\mu \nu}^{i}=f_{4 a j}^{i}\left(A_{\mu}^{4 a} A_{\nu}^{j}-A_{\nu}^{4 a} A_{\mu}^{j}\right), \\
F_{\mu \nu}^{i}=\partial_{\mu} A_{\nu}^{i}-\partial_{\nu} A_{\mu}^{i}+\frac{1}{2} f_{c d j}^{i}\left(A_{\mu}^{c d} A_{\nu}^{j}-A_{\nu}^{c d} A_{\mu}^{j}\right) .
\end{gathered}
$$


The action proposed by MM to describe gravity and supergravity is constructed only in terms of gauge fields, without the tetrad or the spin $3 / 2$ field,

$$
S=\int d^{4} x \varepsilon^{\mu \nu \rho \sigma} \mathcal{F}_{\mu \nu}{ }^{A} \mathcal{F}_{\rho \sigma}{ }^{B} M_{A B}
$$

where $M_{A B}$ is a generalization of the Levi-Civita symbol chosen by MacDowell-Mansouri in the bosonic case and which is defined by

$$
M_{A B}=\left(\begin{array}{cc}
\varepsilon_{a b c d} & 0 \\
0 & i\left(C \gamma_{5}\right)_{i j}
\end{array}\right),
$$

where $\gamma_{5}=i \gamma_{0} \gamma_{1} \gamma_{2} \gamma_{3}, \gamma_{\mu}$ are the Dirac matrices and $C$ is the charge conjugation matrix which satisfies $C \gamma_{5} C^{-1}=\gamma_{5}^{T}$ and $C^{T}=-C$. The above action can be written as

$$
S=\int d^{4} x \varepsilon^{\mu \nu \rho \sigma}\left[\mathcal{F}_{\mu \nu}^{a b} \mathcal{F}_{\rho \sigma}^{c d} \varepsilon_{a b c d}+i \mathcal{F}_{\mu \nu}^{i} \mathcal{F}_{\rho \sigma}^{j}\left(C \gamma_{5}\right)_{i j}\right] .
$$

By identifying $A_{\mu}^{a b} \equiv \omega_{\mu}^{a b}$ with the spin connection, $A_{\mu}^{4 a} \equiv e_{\mu}^{a}$ with the tetrad and $A_{\mu}^{i} \equiv \psi_{\mu}^{i}$ with the gravitino, MM have shown that the action (11) gives the gauge theory of $\mathrm{N}=1$ supergravity, plus cosmological and topological terms [13.

We define the (anti) self-dual part of the field strength (1) as

$$
{ }^{ \pm} \mathcal{F}_{\mu \nu}{ }^{A}=\frac{1}{2}{ }^{ \pm} \mathcal{B}_{B}^{A} \mathcal{F}_{\mu \nu}^{B}
$$

where ${ }^{ \pm} \mathcal{B}_{B}^{A}$ is given by

$$
{ }^{ \pm} \mathcal{B}_{B}^{A}=\left(\begin{array}{cc}
{ }^{ \pm} B_{c d}^{a b} & 0 \\
0 & { }^{ \pm} B_{j}^{i}
\end{array}\right)=\left(\begin{array}{cc}
\frac{1}{2}\left(\delta_{c d}^{a b} \mp i \varepsilon_{c d}^{a b}\right) & 0 \\
0 & \left(1 \pm \gamma_{5}\right)_{j}^{i}
\end{array}\right),
$$

with $\delta_{c d}^{a b}=\delta_{c}^{a} \delta_{d}^{b}-\delta_{d}^{a} \delta_{c}^{b}$. It is then not difficult to see that the self-dual action corresponding to $(9)$ is given by

$$
S^{+}=\int d^{4} x \varepsilon^{\mu \nu \rho \sigma+} \mathcal{F}_{\mu \nu}{ }^{A+} \mathcal{F}_{\rho \sigma}{ }^{B} M_{A B}
$$

which in terms of the $M_{A B}$ components is

$$
S^{+}=\int d^{4} x \varepsilon^{\mu \nu \rho \sigma}\left[+\mathcal{F}_{\mu \nu}^{a b+} \mathcal{F}_{\rho \sigma}^{c d} \varepsilon_{a b c d}+i^{+} \mathcal{F}_{\mu \nu}^{i+} \mathcal{F}_{\rho \sigma}^{j}\left(C \gamma_{5}\right)_{i j}\right] .
$$

This action is the one proposed in [16] as a self dual version of the MM-supergravity gauge theory in terms only on the self-dual spin connection. We will show that similar to YangMills [7,8] and MacDowell-Mansouri theory [11], the linear superposition of (15) and the anti-self-dual corresponding action, does admit a dual description as well. 


\section{DUALITY IN MACDOWELL-MANSOURI GAUGE THEORY OF SUPERGRAVITY}

In this section we show that for a theory of gravity with the structure of a gauge theory and which is also supersymmetric, it is possible to find a dual description [11, 12] given by a supersymmetric non-linear sigma model.

We consider the following action consisting in the linear superposition of the self-dual and anti-self-dual parts of (9)

$$
I=\int d^{4} x \varepsilon^{\mu \nu \rho \sigma}\left({ }^{+} \tau^{+} \mathcal{F}_{\mu \nu}^{A+} \mathcal{F}_{\rho \sigma}^{B}-{ }^{-} \tau^{-} \mathcal{F}_{\mu \nu}^{A-} \mathcal{F}_{\rho \sigma}^{B}\right) M_{A B}
$$

This action can be rewritten as

$$
\begin{aligned}
& I=\int d^{4} x \varepsilon^{\mu \nu \rho \sigma}\left[\left({ }^{+} \tau^{+} \mathcal{F}_{\mu \nu}^{a b+} \mathcal{F}_{\rho \sigma}^{c d}-{ }^{-} \tau^{-} \mathcal{F}_{\mu \nu}^{a b-} \mathcal{F}_{\rho \sigma}^{c d}\right) \varepsilon_{a b c d}\right. \\
& \left.+i\left({ }^{+} \tau^{+} \mathcal{F}_{\mu \nu}^{i}{ }^{+} \mathcal{F}_{\rho \sigma}^{j}-{ }^{-} \tau^{-} \mathcal{F}_{\mu \nu}^{i}-\mathcal{F}_{\rho \sigma}^{j}\right)\left(C \gamma_{5}\right)_{i j}\right] .
\end{aligned}
$$

Substituting (12) and (13) into this action and using the auxiliary formulas

$$
\begin{gathered}
{ }^{ \pm} B_{e f}^{a b}{ }^{ \pm} B_{g h}^{c d} \varepsilon_{a b c d}= \pm 2 i B_{e f g h} \\
{ }^{ \pm} B_{r}^{i \pm} B_{s}^{j}\left(C \gamma_{5}\right)_{i j}= \pm C_{r j} B_{s}^{j}
\end{gathered}
$$

after some computations it is an easy matter to show that the action (16) is equivalent to

$$
I=\frac{1}{2} \int d^{4} x \varepsilon^{\mu \nu \rho \sigma}\left[\left({ }^{+} \tau-{ }^{-} \tau\right) \mathcal{F}_{\mu \nu}^{A} \mathcal{F}_{\rho \sigma}^{B}+\left({ }^{+} \tau+{ }^{-} \tau\right) \tilde{\mathcal{F}}_{\rho \sigma}^{B} \mathcal{F}_{\mu \nu}^{A}\right] M_{A B}
$$

where $\tilde{\mathcal{F}}_{\mu \nu}^{A}$ consist of two parts $\left(\tilde{\mathcal{F}}_{\mu \nu}^{a b}, \tilde{\mathcal{F}}_{\mu \nu}^{i}\right)$ defined by $\tilde{\mathcal{F}}_{\mu \nu}^{a b}=\frac{i}{2} \varepsilon_{c d}^{a b} \mathcal{F}_{\mu \nu}^{c d}$ and $\tilde{\mathcal{F}}_{\mu \nu}^{i}=\gamma_{5 j}^{i} \mathcal{F}_{\mu \nu}^{j}$. The first term in (19) is the MM-supergravity (9). In [11] it was shown that the bosonic part of the second term reduces to the Pontrjagin topological term. The fermionic part of this second term is its corresponding supersymmetric partner.

Now we will find a dual action to (16). In order to do that, as usual, we propose a parent action. For the gauge theory of supergravity of MM we propose this action as follows

$$
L=\int d^{4} x \varepsilon^{\mu \nu \rho \sigma}\left(C_{1}{ }^{+} \mathcal{G}_{\mu \nu}^{A}+\mathcal{G}_{\rho \sigma}^{B}+C_{2}{ }^{-} \mathcal{G}_{\mu \nu}^{A-} \mathcal{G}_{\rho \sigma}^{B}+C_{3}{ }^{+} \mathcal{F}_{\mu \nu}^{A+} \mathcal{G}_{\rho \sigma}^{B}+C_{4}{ }^{-} \mathcal{F}_{\mu \nu}^{A-} \mathcal{G}_{\rho \sigma}^{B}\right) M_{A B}
$$


where $C_{i}$ are constants and ${ }^{ \pm} \mathcal{G}$ are $\operatorname{Osp}(1 \mid 4)$ - Lie algebra valued Lagrange multiplier fields. Integrating out this action with respect to ${ }^{+} \mathcal{G},{ }^{-} \mathcal{G}$ one easily recovers the action (16) with an appropriate choice of the coupling constants $C_{i}$.

In order to get the dual theory we follow the reference [7],8]. Hence, one should start with the partition function

$$
Z=\int \mathcal{D}^{+} \mathcal{G} \mathcal{D}^{-} \mathcal{G} \mathcal{D} \mathcal{A} \exp (-L)
$$

The proposed parent action (20) can be rewritten in terms of the components of $M_{A B}$ in the form

$$
\begin{aligned}
& L=\int d^{4} x \varepsilon^{\mu \nu \rho \sigma}\left[\left(C_{1}+\mathcal{G}_{\mu \nu}^{a b+} \mathcal{G}_{\rho \sigma}^{c d}+C_{2}-\mathcal{G}_{\mu \nu}^{a b-} \mathcal{G}_{\rho \sigma}^{c d}\right) \varepsilon_{a b c d}\right. \\
& +i\left(C_{1}{ }^{+} \mathcal{G}_{\mu \nu}^{i}{ }^{+} \mathcal{G}_{\rho \sigma}^{j}+C_{2}{ }^{-} \mathcal{G}_{\mu \nu}^{i}{ }^{-} \mathcal{G}_{\rho \sigma}^{j}\right)\left(C \gamma_{5}\right)_{i j} \\
& +\left(C_{3}{ }^{+} \mathcal{F}_{\mu \nu}^{a b+} \mathcal{G}_{\rho \sigma}^{c d}+C_{4}{ }^{-} \mathcal{F}_{\mu \nu}^{a b-} \mathcal{G}_{\rho \sigma}^{c d}\right) \varepsilon_{a b c d} \\
& \left.+i\left(C_{3}+\mathcal{F}_{\mu \nu}^{i}+\mathcal{G}_{\rho \sigma}^{j}+C_{4}-\mathcal{F}_{\mu \nu}^{i}-\mathcal{G}_{\rho \sigma}^{j}\right)\left(C \gamma_{5}\right)_{i j}\right]
\end{aligned}
$$

In order to perform the Feynman integral we decompose this functional integration measure $\int \mathcal{D} \mathcal{A}$ into its component fields i.e $\int \mathcal{D} \mathcal{A}_{\mu}^{A}=\int \mathcal{D} A_{\mu}^{a b} \mathcal{D} A_{\mu}^{i} \mathcal{D} A_{\mu}^{4 a}$. Thus we define the dual action $L^{*}$ as follows

$$
\exp \left(-L^{*}\right)=\int \mathcal{D} A_{\mu}^{a b} \mathcal{D} A_{\mu}^{i} \mathcal{D} A_{\mu}^{4 a} \exp (-L)
$$

The partition function written in terms of the dual action reads

$$
Z=\int \mathcal{D}^{+} \mathcal{G} \mathcal{D}^{-} \mathcal{G} \exp \left(-L^{*}\right)
$$

Thus we first integrate out with respect to the field $A_{\mu}^{4 a}$. Before performing the functional integration it is convenient to take into account that the last two rows of action (22) can be rewritten as

$$
\varepsilon^{\mu \nu \rho \sigma}\left(C_{3}{ }^{+} \mathcal{F}_{\mu \nu}^{a b+} \mathcal{G}_{\rho \sigma}^{c d}+C_{4}{ }^{-} \mathcal{F}_{\mu \nu}^{a b-} \mathcal{G}_{\rho \sigma}^{c d}\right) \varepsilon_{a b c d}=-2 i \varepsilon^{\mu \nu \rho \sigma} \mathcal{F}_{\mu \nu}^{a b}\left(C_{3}{ }^{+} \mathcal{G}_{a b \rho \sigma}-C_{4}{ }^{-} \mathcal{G}_{a b \rho \sigma}\right)
$$

and 


$$
i \varepsilon^{\mu \nu \rho \sigma}\left(C_{3}{ }^{+} \mathcal{F}_{\mu \nu}^{i}{ }^{+} \mathcal{G}_{\rho \sigma}^{j}+C_{4}{ }^{-} \mathcal{F}_{\mu \nu}^{i}{ }^{-} \mathcal{G}_{\rho \sigma}^{j}\right)\left(C \gamma_{5}\right)_{i j}=i \varepsilon^{\mu \nu \rho \sigma} \mathcal{F}_{\mu \nu}^{i}\left(C_{3}{ }^{+} \mathcal{G}^{j}{ }_{\rho \sigma}-C_{4}{ }^{-} \mathcal{G}^{j}{ }_{\rho \sigma}\right) C_{i j}
$$

Then the contribution to the field $A_{\mu}^{4 a}$ comes from two pieces of the parent action (22)

$$
\begin{aligned}
& L=\int d^{4} x \varepsilon^{\mu \nu \rho \sigma}\left[\ldots+4 i f_{4 c 4 d}^{a b}\left(C_{3}{ }^{+} \mathcal{G}_{\rho \sigma a b}-C_{4}{ }^{-} \mathcal{G}_{\rho \sigma a b}\right) A_{\mu}^{4 c} A_{\nu}^{4 d}\right. \\
& \left.+2 i f_{4 a k}^{i} A_{\nu}^{k}\left(C_{3}{ }^{+} \mathcal{G}^{j}{ }_{\rho \sigma}-C_{4}{ }^{-} \mathcal{G}^{j}{ }_{\rho \sigma}\right) A_{\mu}^{4 a} C_{i j}+\ldots\right]
\end{aligned}
$$

which can be written as an integral of the gaussian type. The result is

$$
L_{1}^{*}=\int d^{4} x\left[\ldots+A_{\mu}^{i}[\mathbf{T}]_{i j}^{\mu \nu} A_{\nu}^{j}+\ldots\right]+\ln \left(\sqrt{\pi} \operatorname{det} \mathbf{M}^{-\frac{1}{2}}\right)
$$

where

$$
[\mathbf{T}]_{i j}^{\mu \nu}=-\frac{1}{4}[\mathbf{W}]_{c i}^{\alpha \mu}\left[\mathbf{M}^{-1}\right]_{\alpha \beta}^{c d}[\mathbf{W}]_{d j}^{\beta \nu}
$$

with

$$
[\mathbf{W}]_{a j}^{\mu \nu}=2 i \varepsilon^{\mu \nu \rho \sigma} f_{4 a j}^{i}\left(C_{3}{ }^{+} \mathcal{G}_{\rho \sigma}^{k}-C_{4}{ }^{-} \mathcal{G}_{\rho \sigma}^{k}\right) C_{i k}
$$

and

$$
[\mathbf{M}]_{c d}^{\mu \nu}=4 i \varepsilon^{\mu \nu \rho \sigma} f_{4 c 4 d}^{a b}\left(C_{3}{ }^{+} \mathcal{G}_{\rho \sigma a b}-C_{4}{ }^{-} \mathcal{G}_{\rho \sigma a b}\right)
$$

Now we perform the integral $\int \mathcal{D} A_{\mu}^{i}$ of fermionic variables with $i$ being a Majorana index. The terms in the Lagrangian $L_{1}^{*}$ that contribute to the fermionic path integral are

$$
L_{1}^{*}=\int d^{4} x \varepsilon^{\mu \nu \rho \sigma}\left[\ldots+2 i \Theta_{\mu \nu}^{a b}\left(C_{3}{ }^{+} \mathcal{G}_{\rho \sigma a b}-C_{4}{ }^{-} \mathcal{G}_{\rho \sigma a b}\right)+i F_{\mu \nu}^{i}\left(C_{3}{ }^{+} \mathcal{G}_{\rho \sigma}^{j}-C_{4}{ }^{-} \mathcal{G}_{\rho \sigma}^{j}\right) C_{i j}+\ldots\right]
$$

Contribution to the integral over $A_{\mu}^{i}$ from (29) and (33) can be summarized as

$$
L_{1}^{*}=\int d^{4} x\left[\ldots+A_{\mu}^{i}[\mathbf{Z}]_{i j}^{\mu \nu} A_{\nu}^{j}+i \varepsilon^{\mu \nu \rho \sigma}\left(f_{c d j}^{i} A_{\mu}^{c d} \mathcal{H}_{\rho \sigma i}-2 \partial_{\mu} \mathcal{H}_{\rho \sigma j}\right) A_{\nu}^{j}+\ldots\right],
$$

where

$$
[\mathbf{Z}]_{i j}^{\mu \nu}=[\mathbf{T}]_{i j}^{\mu \nu}+[\mathbf{R}]_{i j}^{\mu \nu}
$$


$\mathcal{H}_{\rho \sigma i}=\left(C_{3}{ }^{+} \mathcal{G}_{\rho \sigma}^{j}-C_{4}{ }^{-} \mathcal{G}_{\rho \sigma}^{j}\right) C_{i j}$ and

$$
[\mathbf{R}]_{i j}^{\mu \nu}=i f_{i j}^{a b} \varepsilon^{\mu \nu \rho \sigma}\left(C_{3}{ }^{+} \mathcal{G}_{\rho \sigma a b}-C_{4}{ }^{-} \mathcal{G}_{\rho \sigma a b}\right)
$$

The computation of the fermionic integral is given by

$$
\begin{aligned}
& L_{2}^{*}=\int d^{4} x\left[\ldots+\frac{1}{4} \varepsilon^{\mu \nu \rho \sigma}\left(f_{c d j}^{i} A_{\mu}^{c d} \mathcal{H}_{\rho \sigma i}-2 \partial_{\mu} \mathcal{H}_{\rho \sigma j}\right)\left[\mathbf{Z}^{-1}\right]_{\nu \beta}^{j k} \varepsilon^{\alpha \beta \gamma \delta}\right. \\
& \left.\left(f_{c d k}^{m} A_{\alpha}^{c d} \mathcal{H}_{\gamma \delta m}+2 \partial_{\alpha} \mathcal{H}_{\gamma \delta k}\right)+\ldots\right] \\
& +\ln \left(\sqrt{\pi} \operatorname{det} \mathbf{Z}^{\frac{1}{2}}\right) .
\end{aligned}
$$

Finally we integrate out with respect $A_{\mu}^{a b}$. The terms of the action that contribute to the integration are

$$
\begin{aligned}
& L_{2}^{*}=\int d^{4} x\left[\ldots+\frac{1}{4} \varepsilon^{\mu \nu \rho \sigma} f_{a b j}^{i} \mathcal{H}_{\rho \sigma i}\left[\mathbf{Z}^{-1}\right]_{\nu \beta}^{j k} \varepsilon^{\alpha \beta \gamma \delta} f_{c d k}^{m} \mathcal{H}_{\gamma \delta m} A_{\mu}^{a b} A_{\alpha}^{c d}\right. \\
& \left.-\varepsilon^{\mu \nu \rho \sigma} f_{c d j}^{i} \mathcal{H}_{\rho \sigma i}\left[\mathbf{Z}^{-1}\right]_{\nu \beta}^{j k} \varepsilon^{\alpha \beta \gamma \delta} \partial_{\alpha} \mathcal{H}_{\gamma \delta k} A_{\mu}^{c d}+\ldots\right] .
\end{aligned}
$$

In addition we have the usual contribution coming form the interaction term

$$
L_{2}^{*}=\int d^{4} x \varepsilon^{\mu \nu \rho \sigma}\left[\ldots+2 i F_{\mu \nu}^{a b}\left(C_{3}{ }^{+} \mathcal{G}_{\rho \sigma a b}-C_{4}{ }^{-} \mathcal{G}_{\rho \sigma a b}\right)+\ldots\right]
$$

Before performing the functional integration $\int \mathcal{D} A_{\mu}^{a b}$ with respect to $A_{\mu}^{a b}$ it is convenient to make the following definitions

$$
\begin{gathered}
\mathbf{G}_{c d e f}^{\mu \nu}=i \varepsilon^{\mu \nu \rho \sigma} f_{c d e f}^{a b}\left(C_{3}{ }^{+} \mathcal{G}_{\rho \sigma a b}-C_{4}{ }^{-} \mathcal{G}_{\rho \sigma a b}\right), \\
\mathbf{F}_{a b}^{\mu}=4 i \varepsilon^{\mu \nu \rho \sigma} \partial_{\nu}\left(C_{3}{ }^{+} \mathcal{G}_{\rho \sigma a b}-C_{4}{ }^{-} \mathcal{G}_{\rho \sigma a b}\right), \\
\mathbf{K}_{a b c d}^{\mu \alpha}=\frac{1}{4} \varepsilon^{\mu \nu \rho \sigma} f_{a b j}^{i} \mathcal{H}_{\rho \sigma i}\left[\mathbf{Z}^{-1}\right]_{\nu \beta}^{j k} \varepsilon^{\alpha \beta \gamma \delta} f_{c d k}^{m} \mathcal{H}_{\gamma \delta m}, \\
\mathbf{V}_{c d}^{\mu}=-\varepsilon^{\mu \nu \rho \sigma} f_{c d j}^{i} \mathcal{H}_{\rho \sigma i}\left[\mathbf{Z}^{-1}\right]_{\nu \beta}^{j k} \varepsilon^{\alpha \beta \gamma \delta} \partial_{\alpha} \mathcal{H}_{\gamma \delta k} .
\end{gathered}
$$

The relevant Lagrangian is of the form

$$
L_{2}^{*}=\int d^{4} x\left[\ldots+\left(\mathbf{K}_{a b c d}^{\mu \nu}+\mathbf{G}_{a b c d}^{\mu \nu}\right) A_{\mu}^{a b} A_{\nu}^{c d}+\left(\mathbf{F}_{c d}^{\mu}+\mathbf{V}_{c d}^{\mu}\right) A_{\mu}^{c d}+\ldots\right]
$$


Finally we get the dual action in the sense of [7,8] for the MM gauge theory of supergravity, this is given by

$$
\begin{aligned}
& L^{*}=\int d^{4} x\left[\varepsilon ^ { \mu \nu \rho \sigma } \left(\left(C_{1}{ }^{+} \mathcal{G}_{\mu \nu}^{a b+} \mathcal{G}_{\rho \sigma}^{c d}+C_{2}{ }^{-} \mathcal{G}_{\mu \nu}^{a b-} \mathcal{G}_{\rho \sigma}^{c d}\right) \varepsilon_{a b c d}\right.\right. \\
& +i\left(C_{1}{ }^{+} \mathcal{G}_{\mu \nu}^{i}{ }^{+} \mathcal{G}_{\rho \sigma}^{j}+C_{2}{ }^{-} \mathcal{G}_{\mu \nu}^{i}{ }^{-} \mathcal{G}_{\rho \sigma}^{j}\right)\left(C \gamma_{5}\right)_{i j} \\
& \left.+4 \partial_{\mu} \mathcal{H}_{\rho \sigma j}\left[\mathbf{Z}^{-1}\right]_{\nu \beta}^{j k} \varepsilon^{\alpha \beta \gamma \delta} \partial_{\alpha} \mathcal{H}_{\gamma \delta k}\right) \\
& \left.-\frac{1}{4}\left(\mathbf{F}_{a b}^{\mu}+\mathbf{V}_{a b}^{\mu}\right)\left[(\mathbf{K}+\mathbf{G})^{-1}\right]_{\mu \nu}^{a b c d}\left(\mathbf{F}_{c d}^{\nu}+\mathbf{V}_{c d}^{\nu}\right)\right] \\
& +\ln \left(\pi^{\frac{3}{2}} \operatorname{det} \mathbf{M}^{-\frac{1}{2}} \operatorname{det} \mathbf{Z}^{\frac{1}{2}} \operatorname{det}(\mathbf{K}+\mathbf{G})^{-\frac{1}{2}}\right)
\end{aligned}
$$

It should be remarked that this resulting supersymmetric non-linear sigma model has the same structure of previous models considered in the literature [9].

In the process to get the dual action (44) we have integrated out Feynman integral (21) through the explicit decomposition of the integration measure (23). This choice breaks explicitly its description in the superspace. It has not necessarily to be so. It is interesting to see that the dual action (44) can be also derived completely from the formalism of integration in supermanifolds [17]. We leave the details of this derivation for the appendix A.

\section{DISCUSSION}

In gauge field theory, $S$-duality arises naturally for abelian theories [18]. For non-abelian theories, Seiberg and Witten [5] have shown that supersymmetry is very useful mainly due to the holomorphic properties of the superpotential. Thus, after breaking the gauge symmetry an effective duality can be obtained. For non-supersymmetric gauge theories, a "field theory duality" can be constructed [6], which results in the usual S-duality for the abelian case. However, for non-abelian theories, this dual theory turns out to be a kind of "massive" non-linear sigma model [9]. Of course, $S$-duality is present in the superstring and $\mathrm{M}$ theory approach, with deep non-perturbative consequences. In [19], "gravitational branes" which arise in type II superstrings and M theory have been considered in the search for gravitational duality. 
If one attempts to formulate a program to pursue $S$-duality for gravitation, in the framework of field theories, it seems to us that one should take into account the elements mentioned above for standard field theories.

We need, first, a gauge theory of gravity. This has been done already by MM [13] and other authors [14]. Second, the theory should be supersymmetric. Third, being the MM supergravity a gauge theory, one would need to find matter couplings which should preserve this supersymmetric gauge structure, in such a way that the tetrad and the gravitino field do not appear in the action whole [15]. Fourth, the next step, is to break the symmetry in order to find an "effective gravitational duality".

The third and fourth steps present technical and conceptual challenges. The coupling of matter in these kind of gauge theories of gravity is an open problem [15]. The symmetry breaking, consequently, has to be understood, technically as well as conceptually. Let us remember that we are dealing with a gauge theory of (super)gravity.

In this work, following this program, we have been able to deal with a gauge theory of gravity which is supersymmetric and we were able to find a field theory duality in the sense of references [6]8]. It is interesting to note that our supergroup procedure could also be useful to extend the results for standard Yang-Mills theories to their corresponding supersymmetric versions.

The search to find an effective gravitational $S$-duality in the context of field theory, requires further work and a deeper understanding of the technical and conceptual issues. Work in these aspects is in progress.

\section{Acknowledgments}

This work was supported in part by CONACyT grants 3898P-E9608 and 28454-E. One of us (H.G.-C.) would like to thank CONACyT for support under the program Programa de Posdoctorantes: Estancias Posdoctorales en el Extranjero para Graduados en Instituciones Nacionales 1997-1998 and the Institute for Advanced Study for its hospitality. 


\section{Appendix A. ALternative Derivation OF THE DUAL ACTION}

The purpose of this appendix is to provide an alternative derivation of MM-supergravity dual action (45) from the formalism of integration in supermanifolds [17]. First of all notice that the last two row of Eq. (23) can be written as

$$
A_{\mathcal{F}}=-2 i \mathcal{F}_{\mu \nu}^{a b} \mathcal{H}_{a b}^{\mu \nu}+i \mathcal{F}_{\mu \nu}^{i} \mathcal{H}_{i}^{\mu \nu}
$$

where $\mathcal{H}_{a b}^{\mu \nu}=\varepsilon^{\mu \nu \rho \sigma}\left(C_{3}{ }^{+} \mathcal{G}_{\rho \sigma a b}-C_{4}{ }^{-} \mathcal{G}_{\rho \sigma a b}\right)$ and $\mathcal{H}_{i}^{\mu \nu}=\varepsilon^{\mu \nu \rho \sigma}\left(C_{3}{ }^{+} \mathcal{G}_{\rho \sigma i}-C_{4}{ }^{-} \mathcal{G}_{\rho \sigma i}\right)$, with ${ }^{ \pm} \mathcal{G}_{\mu \nu i}=$ $C_{i j}{ }^{ \pm} \mathcal{G}_{\mu \nu}^{j}$.

Taking into account the definition of the field strength $\mathcal{F}_{\mu \nu}^{A}$, after partial integrations we get

$$
\begin{aligned}
A_{\mathcal{F}}= & -K_{a b}^{\mu \nu} A_{\mu}^{a} A_{\nu}^{b}-2 W_{a i}^{\mu \nu} A_{\mu}^{a} A_{\nu}^{i}-R_{i j}^{\mu \nu} A_{\mu}^{i} A_{\nu}^{j}-G_{a b c d}^{\mu \nu} A_{\mu}^{a b} A_{\nu}^{c d} \\
& +U_{a b i}^{\mu \nu} A_{\mu}^{a b} A_{\nu}^{i}+4 i \partial_{\mu} \mathcal{H}_{a b}^{\mu \nu} A_{\nu}^{a b}-2 i \partial_{\mu} \mathcal{H}_{i}^{\mu \nu} A_{\nu}^{i},
\end{aligned}
$$

where $A_{\mu}^{a}=A_{\mu}^{4 a}$ and $K_{a b}^{\mu \nu}=4 i f_{4 a 4 b}^{c d} \mathcal{H}_{c d}^{\mu \nu}, W_{a i}^{\mu \nu}=i f_{4 a i}^{j} \mathcal{H}_{j}^{\mu \nu}, R_{i j}^{\mu \nu}=i f_{i j}^{a b} \mathcal{H}_{a b}^{\mu \nu}, G_{a b c d}^{\mu \nu}=i f_{a b c d}^{e f} \mathcal{H}_{e f}^{\mu \nu}$ and $U_{a b i}^{\mu \nu}=i f_{a b i}^{j} \mathcal{H}_{j}^{\mu \nu}$.

This quadratic form can be diagonalized by defining the $\tilde{A}$-variables

$$
\begin{aligned}
A_{\mathcal{F}}= & -K_{a b}^{\mu \nu} \tilde{A}_{\mu}^{a} \tilde{A}_{\nu}^{b}+\frac{1}{4} Z_{i j}^{\mu \nu} \tilde{A}_{\mu}^{i} \tilde{A}_{\nu}^{j}-J_{a b c d}^{\mu \nu} \tilde{A}_{\mu}^{a b} \tilde{A}_{\nu}^{c d}-4 J_{\mu \nu}^{-1}{ }_{\mu b c d} \partial_{\rho} \mathcal{H}_{a b}^{\rho \mu} \partial_{\sigma} \mathcal{H}_{c d}^{\sigma \nu} \\
& +N_{\mu \nu}^{i j} \partial_{\rho} \mathcal{H}_{i}^{\rho \mu} \partial_{\sigma} \mathcal{H}_{j}^{\sigma \nu}+8 J^{-1}{ }_{\mu \nu}^{a b c d} U_{c d j}^{\nu \theta} Z_{\theta \tau}^{-1}{ }_{\theta \rho}^{j i} \partial_{\rho} \mathcal{H}_{a b}^{\rho \mu} \partial_{\sigma} \mathcal{H}_{i}^{\sigma \tau},
\end{aligned}
$$

where

$$
\begin{aligned}
& Z_{i j}^{\mu \nu}=4\left(-R_{i j}^{\mu \nu}+W_{a i}^{\rho \mu} K_{\rho \sigma}^{-1}{ }_{\rho \sigma}^{a b} W_{b j}^{\sigma \nu}\right), \\
& J_{a b c d}^{\mu \nu}=G_{a b c d}^{\mu \nu}-U_{a b i}^{\mu \rho} Z_{\rho \sigma}^{-1 i j} U_{c d j}^{\nu \sigma}, \\
& N_{\mu \nu}^{i j}=-4\left(Z_{\mu \nu}^{-1 i j}+Z_{\sigma \mu}^{-1} U_{c d l}^{i j} J_{\tau \theta}^{-1}{ }_{\tau \theta}^{a b c d} U_{a b k}^{\tau \rho} Z_{\rho \nu}^{-1 k j}\right)
\end{aligned}
$$

and

$$
\begin{aligned}
& \tilde{A}_{\mu}^{a}=A_{\mu}^{a}+K_{\mu \rho}^{-1 a b} W_{b i}^{\rho \sigma} A_{\sigma}^{i}, \\
& \tilde{A}_{\mu}^{i}=A_{\mu}^{i}+2 Z_{\mu \nu}^{-1}{ }_{\mu \nu}^{i j}\left(2 i \partial_{\rho} \mathcal{H}_{j}^{\rho \nu}-U_{a b j}^{\rho \nu} A_{\rho}^{a b}\right), \\
& \tilde{A}_{\mu}^{a b}=A_{\mu}^{a b}+2 i J_{\mu \nu}^{-1 a b c d}\left(\partial_{\rho} \mathcal{H}_{c d}^{\rho \nu}-U_{c d i}^{\nu \rho} Z_{\rho \sigma}^{-1}{ }^{i j} \partial_{\tau} \mathcal{H}_{j}^{\tau \sigma}\right) .
\end{aligned}
$$

Now, if we define the matrix 


$$
\mathcal{M}=\left(\begin{array}{cc}
G & U^{T} \\
U & Z
\end{array}\right)
$$

whose inverse is given by (see for example [17])

$$
\left(\begin{array}{cc}
\left(1-G^{-1} U^{T} Z^{-1} U\right)^{-1} G^{-1} & -\left(1-G^{-1} U^{T} Z^{-1} U\right)^{-1} G^{-1} U^{T} Z^{-1} \\
-\left(1-Z^{-1} U G^{-1} U^{T}\right)^{-1} Z^{-1} U G^{-1} & \left(1-Z^{-1} U G^{-1} U^{T}\right)^{-1} Z^{-1}
\end{array}\right)
$$

and we take into account the identity

$$
Z^{-1} U^{T} J^{-1} U=1-\left(1-Z^{-1} U^{T} G^{-1} U\right)^{-1}
$$

we obtain, written in a matrix formulation

$$
A_{\mathcal{F}}=-\tilde{A}^{T} K \tilde{A}-\frac{1}{4} \tilde{A}^{T} Z \tilde{A}-\tilde{A}^{T} J \tilde{A}+4 \mathcal{M}_{\mu \nu}^{-1} A B \partial_{\rho} \mathcal{H}_{B}^{\rho \nu} \partial_{\sigma} \mathcal{H}_{A}^{\sigma \mu}
$$

In order to integrate the components of $A_{\mu}^{A}$, we must first make the change of variables (A.5), it mixes the variables among them and the corresponding jacobian should to be taken into account. Due to the fact that bosonic and fermionic variables come into play, the jacobian is given by a superdeterminant, defined by [17]

$$
\operatorname{Sdet}\left(\begin{array}{cc}
A & C \\
D & B
\end{array}\right)=\frac{\operatorname{det}\left(A-C B^{-1} D\right)}{\operatorname{det} B},
$$

¿From (A.5), it can be seen that the superdeterminant of the matrix of the homogeneous part of the transformation is 1 . Thus, there is no contribution from the jacobian.

The integrations to be done are gaussian. Hence the result will be the product of determinants $\operatorname{det} K^{-\frac{1}{2}} \operatorname{det} J^{-\frac{1}{2}} \operatorname{det} Z^{\frac{1}{2}}$, where the last one has a positive power because it comes from a fermionic integral. From the definition of the matrix $J=G\left(1-G^{-1} U Z^{-1} U^{T}\right)$, we see that the product of the last two determinants can be written as the superdeterminant of the matrix (A.6).

Thus we get that the dual action for MM-supergravity is given by

$$
\begin{aligned}
& L^{*}=\int d^{4} x \varepsilon^{\mu \nu \rho \sigma}\left(C_{1}{ }^{+} \mathcal{G}_{\mu \nu}^{A}+\mathcal{G}_{\rho \sigma}^{B}+C_{2}{ }^{-} \mathcal{G}_{\mu \nu}^{A}-\mathcal{G}_{\rho \sigma}^{B}\right) M_{A B}+4 \mathcal{M}^{-1}{ }_{\mu \nu}^{A B} \partial_{\rho} \mathcal{H}_{B}^{\rho \nu} \partial_{\sigma} \mathcal{H}_{A}^{\sigma \mu} \\
& +\ln \left(\operatorname{det} K^{-\frac{1}{2}} \operatorname{Sdet} \mathcal{M}^{-\frac{1}{2}}\right)
\end{aligned}
$$

Writing in components this action is, of course, equivalent to the action (44). 


\section{REFERENCES}

[1] L. Alvarez-Gaume and F. Zamora, "Duality in Quantum Field Theory (and String Theory)", hep-th/9709180. W. Lerche, Nucl. Phys. Proc. Suppl. 55B (1997) 83.

[2] J.H. Schwarz, Nucl. Phys. Proc. Suppl. 55B (1997) 1; P.K. Townsend, "Four Lectures on M-theory", hep-th/9612121; A. Sen, "An Introduction to Non-perturbative String Theory", hep-th/9802051.

[3] For the role and interesting properties of non-BPS states see, M. Strassler, J. High Energy Phys. 9809 (1998) 017; "On Phases of Gauge Theories and the Role of NonBPS Solitons in Field Theory", hep-th/9808073; J. Blum and K. Dienes, Nucl. Phys, B516 (1998) 83; Nucl. Phys. B 520 93; A. Sen, J. High Energy Phys. 06 (1998) 007; J. High Energy Phys. 9808 (1998) 010.

[4] S. Kachru and E. Silverstein, Phys. Rev. Lett. 804855 (1998).

[5] N. Seiberg and E. Witten, Nucl. Phys. B426, 19 (1994) ; N. Seiberg and E. Witten, Nucl. Phys. B431, 484 (1994).

[6] Y. Lozano, Phys. Lett. B 364, 19 (1995); A.A. Kehagias, "A Canonical Approach to S-Duality in Abelian Gauge Theory", hep-th/9508159.

[7] O. Ganor and J. Sonnenschein, Int. J. Mod. Phys. A 115701 (1996).

[8] N. Mohammedi, "Classical Duality in Gauge Theories", hep-th/9507040.

[9] D.Z. Freedman and P.K. Townsend, Nucl Phys. B 177, 282 (1981); P.C. West, Phys. Lett. B 76, 569 (1978); A.H. Chamseddine, Ann. Phys. 113, 212 (1978); S. Gotzes and A.C. Hirshfeld, Ann. Phys. (N.Y.) 203, 410 (1990).

[10] H. García-Compeán, O. Obregón, J.F. Plebański and C. Ramírez, Phys. Rev. D 57 (1998) 7501.

[11] H. García-Compeán, O. Obregón and C. Ramírez, Phys. Rev. D 58 (1998) 104012. 
[12] H. García-Compeán, O. Obregón and C. Ramírez, "Pursuing Gravitational S-Duality", to appear in Chaos, Solitons and Fractals (1999), hep-th/9807188.

[13] S.W. MacDowell and F. Mansouri, Phys. Rev. Lett. 38, 739 (1997); F. Mansouri, Phys. Rev. D 16, 2456 (1977).

[14] H.R. Pagels, Phys. Rev. D 29, 1690 (1984).

[15] F. Wilczek, Phys. Rev. Lett 80, 4851 (1998).

[16] J.A. Nieto, O. Obregón and J. Socorro, Phys. Rev. D 50, R3583 (1994); J.A. Nieto, J. Socorro and O. Obregón, Phys. Rev. Lett. 76, 3482 (1996).

[17] B. DeWitt, Supermanifolds, Cambridge University Press, 1984.

[18] E. Witten, Selecta Mathematica, New Series, 1, 383 (1995).

[19] C.M. Hull, Nucl. Phys. B 509 (1998) 216. 